\title{
How Patients Use a Patient Portal: An Institutional Case Study of Demographics and Usage Patterns
}

\author{
Raymond Tsai ${ }^{1}$ Elijah J. Bell III', Hawkin Woo ${ }^{1,3}$ \\ ${ }^{1}$ Department of Health Information Technology, University of \\ California, Los Angeles, California, United States \\ 2 Department of Emergency Medicine, David Geffen School of \\ Medicine at UCLA, Los Angeles, California, United States \\ 3 Department of Medicine, David Geffen School of Medicine at UCLA, \\ Los Angeles, California, United States \\ Appl Clin Inform 2019;10:96-102.
}

\author{
Kevin Baldwin ${ }^{1}$ Michael A. Pfeffer ${ }^{1,3}$
}

\begin{abstract}
Address for correspondence Raymond Tsai, MD, MS, Department of Health Information Technology, University of California, Los Angeles, 13646 Highway 33, Lost Hills, CA 93249, United States (e-mail: raymondtsai@post.harvard.edu).
\end{abstract}

\section{Abstract}

Keywords

- electronic health records

- patient portal

- patient engagement

- medical history
Background Given the widespread electronic health record adoption, there is increasing interest to leverage patient portals to improve care.

Objective To determine characteristics of patient portal users and the activities they accessed in the patient portal.

Methods We performed a retrospective analysis of patient portal usage at University of California, Los Angeles, Health from July 2014 to May 2015. A total dataset of 505,503 patients was compiled with 396,303 patients who did not register for the patient portal and 109,200 patients who registered for a patient portal account. We compared patients who did not register for the online portal to the top 75th percentile of users based on number of logins, which was done to exclude those who only logged in to register. Finally, to avoid doing statistical analysis on too large of a sample and overpower the analysis, we performed statistical tests on a random sample of 300 patients in each of the two groups.

Results Patient portal users tended to be older (49.45 vs. 46.22 years in the entire sample, $p=0.008$ in the random sample) and more likely female (62.59 vs. $54.91 \%$ in the entire sample, $p=0.035$ in the random sample). Nonusers had more monthly emergency room (ER) visits on average $(0.047$ vs. $0.014, p<0.001)$. The most frequently accessed activity on the portal was viewing laboratory results $(79.7 \%$ of users looked at laboratory results).

Conclusion There are differences between patient portal users and nonusers, and further understanding of these differences can serve as foundation for further investigation and possible interventions to drive patient engagement and health outcomes.

\section{Background and Significance}

In the last several years, there has been a surge of adoption of health information technology and electronic health records (EHRs). The most updated numbers from the Office of the National Coordinator for Health Information Technology reported that in $2015,83.8 \%$ of nonfederal acute care hospitals had a basic EHR system, up from $44.4 \%$ in $2012 .{ }^{1}$ This trend is mirrored in office-based physician practices. ${ }^{2,3}$

received

September 24, 2018

accepted after revision

December 10, 2018
Along with this increase in EHR adoption comes a corresponding increase in EHR functionality available to patients for patient engagement. ${ }^{4}$

While engaging patients is widely accepted as a way to improve care, it is unclear that patient portals are the way to achieve this goal. ${ }^{5}$ Although there have been cases that have shown improved care through use of patient portals, thus far the benefits of patient portals have been mixed. $^{6-11}$
License terms Stuttgart · New York

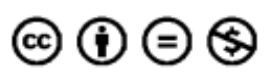


One reason for potential gaps in demonstrating improved care is that despite encouraging enrollment into patient portals, little is known about how patients interact with these portals. ${ }^{12}$ Most of what is known about patients' use of portals come from self-reported patient attitudes and expectations. ${ }^{13-16}$ Contrastingly, other industries have used consumer-based data analytics to improve their products. ${ }^{17}$ We seek to further illuminate characteristics associated with our users and how they interact with the portal.

\section{Objective}

The goal of this project was to identify characteristics of patients who registered for and logged in to the patient portal, and to summarize activities patients engage in when they accessed the portal. This would assist organizations in prioritizing resources to strategically optimize the portal and increase patient engagement.

\section{Methods}

A retrospective analysis of patient portal usage was performed at the University of California, Los Angeles (UCLA) Health, an urban, tertiary care health system comprising outpatient clinics and four hospitals with a combined total of 805 inpatient beds and 693,555 outpatient clinic visits (not including the community-based primary care network) in
$2015 .^{18}$ Data were examined from July 1, 2014 to May 30, 2015. Demographic data, patient portal usage, and health care utilization data were collected for each patient portal user (- Table 1).

All data were extracted using structured query language (SQL) from the EHR database (Epic Systems, Inc.) and were merged using the medical record number (MRN) to form a single dataset for analysis using Stata 14 (StatCorp LLC). After merging, MRNs were deleted and each unique patient was assigned a sequential identifier. Inclusion criteria consisted of all existing patient portal accounts and all patients who received an invitation to activate their account but did not have an active account. Exclusion criteria were patients' accounts that were activated after February 1, 2015, those that had only page view or edits without logins, and those without demographic data available (-Fig. 1).

The top 75th percentile for logins was used as the criteria for active users (above 0.25 logins per month, or $\sim 1$ login every 4 months) to exclude those who only logged in very rarely or only to create an account. This was done to more accurately represent a sample of active users. Overall demographic data are included in -Table 2 .

Given the large dataset, we performed an a priori power analysis using $G^{*}$ Power 3.1 software and determined the appropriate sample size to detect a mean average age difference of 5 years with a power of 0.8 was approximately 300 per group, so we used a random total sample of 600 (300 active users and 300 without a portal account). Without

Table 1 List of variable queried from database

\begin{tabular}{|c|c|}
\hline $\begin{array}{l}\text { Data used to characterize MyChart Use } \\
\text { (each of the listed data points had } \\
11 \text { separate variables to represent counts } \\
\text { each month from July } 2014 \text { to May 2015) }\end{array}$ & $\begin{array}{l}\text { Demographic data per MyChart user } \\
\text { (generated June } 20,2015 \text { ) }\end{array}$ \\
\hline \multirow{5}{*}{$\begin{array}{l}\text { 1. Login count } \\
\text { 2. Appointments requested } \\
\text { 3. Appointments cancelled } \\
\text { 4. Current Health Issues page viewed } \\
\text { 5. Current Health issues updated by user } \\
\text { 6. Medications page viewed } \\
\text { 7. Medication update sent } \\
\text { 8. Allergies page viewed } \\
\text { 9. Allergies updated by user } \\
\text { 10. Immunization page seen } \\
\text { 11. Health summary page seen } \\
\text { 12. Medical history page seen } \\
\text { 13. Hospital admission summary page seen } \\
\text { 14. Health trends page seen } \\
\text { 15. Letters page seen } \\
\text { 16. Letters printed } \\
\text { 17. Who's accessed the record viewed } \\
\text { 18. Family records viewed } \\
\text { 19. Contact information updated } \\
\text { 20. Laboratory results viewed } \\
\text { 21. Account holder is proxy for another } \\
\text { 22. Proxy is on the account } \\
\text { 23. Wallet card viewed (or printed) } \\
\text { 24. Wallet card edited } \\
\text { 25. Mobile device logins }\end{array}$} & $\begin{array}{l}\text { 1. Age } \\
\text { 2. Gender } \\
\text { 3. Race } \\
\text { 4. Primary language } \\
\text { 5. Insurance carrier } \\
\text { 6. Alive or deceased }\end{array}$ \\
\hline & $\begin{array}{l}\text { Health care utilization per MyChart user } \\
\text { (each of the listed data points had } 11 \text { separate variables to represent } \\
\text { counts each month from July } 2014 \text { to May 2015) }\end{array}$ \\
\hline & $\begin{array}{l}\text { 1. Number of outpatient clinic visits } \\
\text { 2. Number of in-patient admission } \\
\text { 3. Number of Emergency room (ER) visits } \\
\text { 4. Number of no-shows }\end{array}$ \\
\hline & $\begin{array}{l}\text { Patient portal enrollment } \\
\text { (generated on May 29, 2015) }\end{array}$ \\
\hline & 1. Month and year of enrollment \\
\hline
\end{tabular}




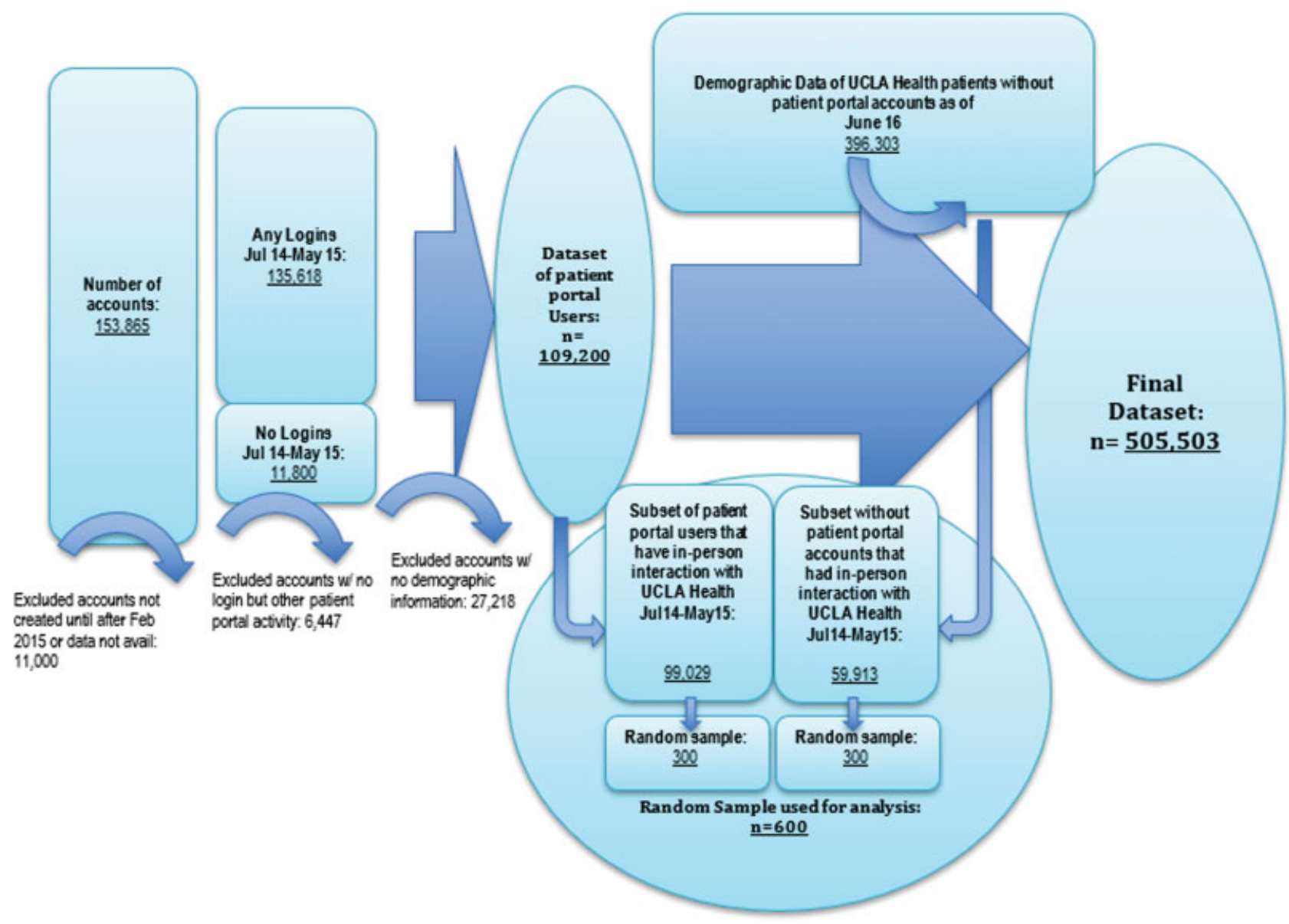

Fig. 1 Summary of data flow to create datasets for analysis.

taking this step, the large sample size would generate such a high power for each statistical test that every difference would be statistically significant regardless of magnitude. Stata was used to generate the random sample. Wilcoxon rank sum tests were used for continuous nonparametric data and chi-squared tests were used for categorical data. To exclude patients that did not interact with UCLA Health during the study period, we limited the sampling to those who had any in-person encounter from the same period of July 1, 2014 to May 30, 2015.

Finally, we looked at how all users interacted with the system using page loads for each page of the patient portal. We looked at the percent of users who accessed certain parts of the portal at least once during the study period. UCLA was not part of the OpenNotes initiative at the time of study. Messaging through the portal was not examined. For the number of logins, we took the average per user per month, only using the number of months a patient was enrolled in the portal during the study period in the denominator.

\section{Results}

We identified 109,200 patients who met inclusion criteria as patient portal users. The top 75th percentile of users accounted for 82,541 patients, who we used to represent active users. The overall characteristics of patients without a patient portal account compared with patient portal users and an analysis on the random sample $(n=600)$ comparing those without a portal account to active users are presented in - Table 2. The activities of the patient portal that were most used are presented in - Table 3. Overall, the data can be summarized in terms of demographic data, health care utilization, and patient portal usage.

\section{Demographic Data}

Patients who were active portal users were on average older ( 49.45 vs. 46.22 years old) and female ( 62.59 vs. $54.91 \%$ ). Both the differences in mean age $(p=0.008)$ and gender $(p=0.035)$ were significant in the random sample. It is also notable that there was a bimodal peak in terms of active users, with active users more likely to be in their 30s and 60s. The difference amongst age groups was also found to be significant $(p<0.001)$. Differences in racial composition, insurance, and language were not found to be significant in this population.

\section{Health Care Utilization}

There were significant differences in health care utilization between those without a patient portal account and active users. Data are only presented on the random sample, which consisted of those who had either an inpatient or outpatient face to face interaction with our health system. Since we did 
How Patients Use a Patient Portal Tsai et al. 99

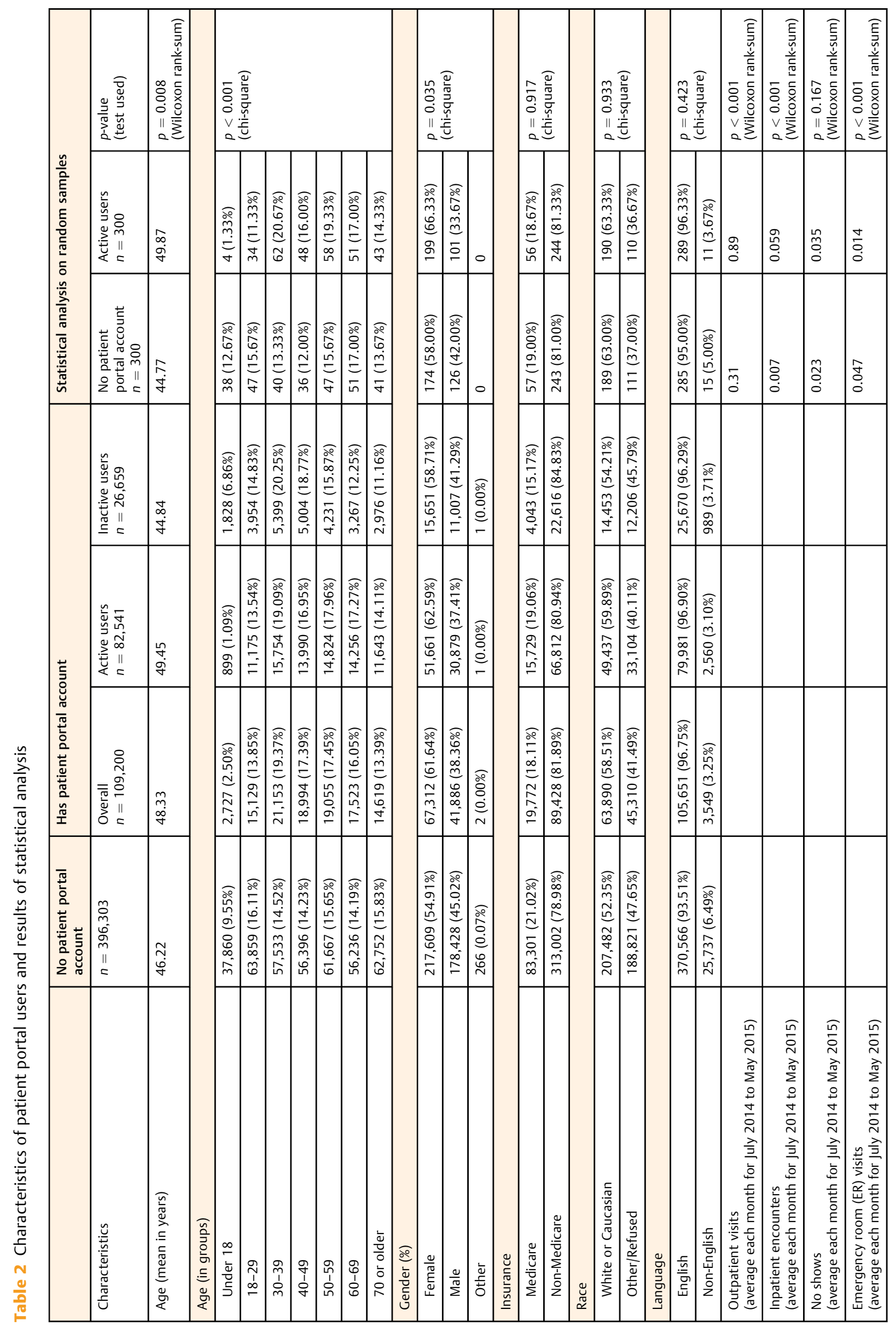


Table 3 Patient portal usage between July 2014 and May 2015

\begin{tabular}{|l|l|}
\hline Patient portal usage metric & $\begin{array}{l}\text { Overall } \\
\boldsymbol{n}=109,200\end{array}$ \\
\hline Logged in (\%) & $100,782(92.29)$ \\
\hline Logins (average per user per month) & 1.92 \\
\hline Mobile users (\%) & $10,503(9.62)$ \\
\hline $\begin{array}{l}\text { Logins using mobile device (average } \\
\text { per user per month) }\end{array}$ & 0.22 \\
\hline Requested an appointment (\%) & $13,885(12.72)$ \\
\hline Cancelled an appointment (\%) & $10,548(9.66)$ \\
\hline Viewed health issues (\%) & $17,262(15.81)$ \\
\hline Updated health issues (\%) & $1,240(1.14)$ \\
\hline Viewed medications (\%) & $57,972(53.09)$ \\
\hline Requested medication update (\%) & $2,032(1.86)$ \\
\hline Viewed allergies (\%) & $54,499(49.91)$ \\
\hline Updated allergies (\%) & $475(0.43)$ \\
\hline Viewed immunizations page (\%) & $55,717(51.02)$ \\
\hline Viewed health summary (\%) & $48,055(44.01)$ \\
\hline Viewed Medical History (\%) & $35,349(32.37)$ \\
\hline Viewed hospital admission summary (\%) & $22,811(20.89)$ \\
\hline Viewed health trends (\%) & $18,277(16.74)$ \\
\hline Viewed letters (\%) & $24,142(22.11)$ \\
\hline Printed letter (\%) & $8,920(8.17)$ \\
\hline Viewed audit of who accessed records & $24,927(22.83)$ \\
\hline Viewed laboratory results & $87,030(79.7)$ \\
\hline Proxy assigned to account & $703(0.6)$ \\
\hline Updated contact info & $1,064(0.9)$ \\
\hline
\end{tabular}

not have access to claims, we cannot say whether patients had other interactions with other local health systems or ERs. Patients without a portal account had on average fewer outpatient visits per month $(0.31$ vs. $0.89, p<0.001)$ and fewer inpatient visits per month (0.007 vs. $0.059, p<0.001)$, but had more ER visits per month in our health system than patients who were active with the portal (0.047 vs. 0.014 , $p<0.001)$. The difference between no-show appointments was not significant.

\section{Patient Portal Usage}

Data on the use of the patient portal activities show which features were accessed by those who had a patient portal account. Overall, 92.29\% of patients with a portal account logged in at least once; however, this is likely inflated by logins to establish an account. On average, there were 1.92 logins per user per month. The distribution of data was skewed with some users logging in multiple times, which could also happen if the login times out. 9.62\% used their mobile device to login at least once. The most frequently accessed activity when logging in was viewing laboratories, which was done by $79.7 \%$ of users. Updating allergies was the activity that occurred the least, with only $0.43 \%$ of users doing so. Similarly, other activities that would allow patients to update information such as contact information, health issues, or medications were accessed infrequently. Patients did use the portal for requesting and cancelling appointments, with these features being used by 12.72 and $9.66 \%$ of patients, respectively.

\section{Discussion}

This case study analysis showing patient portal user characteristics and usage patterns was important for our health system. Prior to this project, this level of data was not compiled for regular use. This project laid the foundation for increased access to information by our patient portal development team to inform their activities. For organizations that do not have these data readily available, this case study can be used as an example of one organization's initial query into patient portal usage data.

For our patient population, some differences were noted in demographic characteristics which warrant further exploration. There are more users in their 30 s, which could be attributed to this age group's ease with technology and higher birth rate for women aged 30 to 34 than for women aged 25 to $29 .{ }^{19}$ However, there is another peak of users in their $60 \mathrm{~s}$, which is likely driven by higher health care utilization. This bimodal pattern has been reported by others, but some studies also show higher portal use among younger patients. ${ }^{12,20}$ In future analysis, we should also extract data to adjust for utilization and medical complexity. In addition, although viewing laboratories is the most accessed feature on the site as demonstrated by other studies, ${ }^{21}$ there should be further exploration as to why other parts of the patient portal are not used. Other features of the portal may be considered low value to patients, in which case we should invest less in those areas, or on the contrary, they could be poorly designed. Focus groups would be a way to gather more information on what is driving observed usage patterns in different age groups and subpopulations. Some projects have done such focus groups, and one project has also tailored staff and patient training to improve usage. $^{22-25}$ Although there were no significant differences in language and race, we would want to ensure accurate capture of demographic and social information, such as education level, before drawing conclusions about disparities (or lack thereof) in patient portal use.

There were differences in health care utilization between active users and nonusers of the patient portal. Patients who are active users of the portal had more outpatient and inpatient visits and fewer ER visits. Increased inpatient utilization in our data is congruent with previous findings of increased odds of readmission among active portal users, $^{26}$ though we did not longitudinally link visits in our analysis to be able to determine if this is also reflected in our sample. A potential next step is to associate patient portal utilization with metrics of quality such as readmission rates.

It is important to note that there is likely significant confounding between outpatient visits, inpatient visits, and ER visits, and to draw any conclusions, an analysis would 
need to adjust for these interactions. For instance, those who are more engaged with outpatient and inpatient visits would likely use the patient portal more, and similarly would also have less need to engage in ER visits. A more careful design would be needed to attribute the driving force for fewer ER visits to patient portal use.

The main limitation of our study is the cross-sectional analysis used. Given the prior lack of information on patient portal users for our organization, we started with a basic analysis to explore what areas could be further investigated. However, this analysis was not in depth enough to draw strong conclusions or causal relationships. We suspect many other organizations may be in a similar situation. In addition, future studies should look at time spent on different areas of the patient portal instead of clicks as a better clue to which sections are engaging to patients.

Ultimately, we should be driving toward improved health outcomes through patient engagement using the patient portal. Further investigations to see if certain usage patterns and features of the portal could be associated with better health outcomes are needed.

\section{Conclusion}

There are differences between patient portal users and nonusers and these differences can be used to help drive portal development. This adds to the growing literature of patient portal users, including demographics, medical utilization, and patient portal utilization. Taken together, these studies can serve as the foundation for further investigation and possible interventions to drive patient engagement for improved health outcomes.

\section{Clinical Relevance Statement}

There are limited data on patient portal users despite encouraging implementation of patient portals in multiple organizations. This case study can help others better understand patient portal users until they perform their own analysis of users. Finally, organizations can use this example to help inform their own investigations of patient portal users in terms of potential variables, including new variables or study designs needed for further analysis.

\section{Multiple Choice Questions}

1. This organization's patient portal users are different than nonusers in:
a. Language.
b. Ethnicity.
c. Emergency room utilization.
d. No-show rate.

Correct Answer: The correct answer is option c. Differences in language, ethnicity, and number of average monthly no-show appointments between patient portal users and nonusers were not found to be significant within this organization's analysis.
2. The analysis used in this case study most resembles what form of study design:

a. Cross-sectional study.

b. Longitudinal study.

c. Regression analysis for predictive analytics.

d. Case-control study.

Correct Answer: The correct answer is option a. As stated in the discussion, this is a cross-sectional study because it looked at the characteristics of a population at a given point in time.

\section{Protection of Human and Animal Subjects}

The institutional review board (IRB) examined our study and determined it was exempt.

\section{Funding}

Dr. Bell's effort was supported in part by the NIH National Center for Advancing Translational Science (NCATS), UCLA CTSI Grant Number TL1TR001883.

\section{Conflict of Interest}

None declared.

\section{References}

1 Henry J, Pylypchuk Y, Searcy T, Patel V. Adoption of electronic health record systems among US non-federal acute care hospitals: 2008-2015. ONC Data Brief. 2016;35:1-9. Available at: https:// www.healthit.gov/sites/default/files/briefs/2015_hospital_adoption_db_v17.pdf. Accessed December 27, 2018

2 Jamoom EW, Yang N, Hing E. Adoption of certified electronic health record systems and electronic information sharing in physician offices: United States, 2013 and 2014. NCHS Data Brief 2016;(236):1-8

3 Jamoom E, Yang N. Table of electronic health record adoption and use among office-based physicians in the U.S., by state: 2015 National Electronic Health Records Survey. Available at: https:// www.cdc.gov/nchs/data/ahcd/nehrs/2015_nehrs_web_table.pdf. Published 2016. Accessed December 27, 2018

4 Henry J, Pylypchuk Y, Patel V. Electronic capabilities for patient engagement among US non-federal acute care hospitals: 20122015. The Office of the National Coordinator for Health Information Technology. 2016. Available at: https://dashboard.healthit. gov/evaluations/data-briefs/hospitals-patient-engagement-electronic-capabilities-2015.php. Accessed December 27, 2018

5 Leventhal R. Survey: portals aren't enough for successful patient engagement. Healthcare Informatics Magazine. Available at: https://www.healthcare-informatics.com/news-item/himss-analytics-survey-portals-aren-t-enough-engage-patients. Accessed October 26, 2018

6 Henry SL, Shen E, Ahuja A, Gould MK, Kanter MH. The Online Personal Action Plan: a tool to transform patient-enabled preventive and chronic care. Am J Prev Med 2016;51(01):71-77

7 Ammenwerth E, Schnell-Inderst P, Hoerbst A. The impact of electronic patient portals on patient care: a systematic review of controlled trials. J Med Internet Res 2012;14(06):e162

8 Goldzweig CL, Orshansky G, Paige NM, et al. Electronic patient portals: evidence on health outcomes, satisfaction, efficiency, and attitudes: a systematic review. Ann Intern Med 2013;159(10):677-687

9 Kruse CS, Argueta DA, Lopez L, Nair A. Patient and provider attitudes toward the use of patient portals for the management of chronic disease: a systematic review. J Med Internet Res 2015; 17(02): 40

10 Murphy-Abdouch K, Dolezel D, McLeod A. Patient access to personal health information: an analysis of the consumer's 
perspective. Perspect Health Inf Manag 2017. Available at: http:// perspectives.ahima.org/wp-content/uploads/2018/06/PatientAccesstoPHI.pdf. Accessed December 27, 2018

11 Health Affairs. Lessons from more than a decade in patient portals. Available at: http://www.healthaffairs.org/do/10.1377/ hblog20160407.054362/full/. Accessed October 26, 2018

12 Powell KR. Patient-perceived facilitators of and barriers to electronic portal use: a systematic review. Comput Inform Nurs 2017; 35(11):565-573

13 Jones JB, Weiner JP, Shah NR, Stewart WF. The wired patient: patterns of electronic patient portal use among patients with cardiac disease or diabetes. J Med Internet Res 2015;17(02):e42

14 Clark SJ, Costello LE, Gebremariam A, Dombkowski KJ. A national survey of parent perspectives on use of patient portals for their children's health care. Appl Clin Inform 2015;6(01):110-119

15 Gordon NP, Hornbrook MC. Differences in access to and preferences for using patient portals and other eHealth technologies based on race, ethnicity, and age: a database and survey study of seniors in a large health plan. J Med Internet Res 2016;18(03):e50

16 Moll J, Rexhepi H, Cajander Å, et al. Patients' experiences of accessing their electronic health records: National Patient Survey in Sweden. J Med Internet Res 2018;20(11):e278

17 Barton D, Court D. Making advanced analytics work for you. Harv Bus Rev 2012;90(10):78-83

18 About Us. Best Healthcare, Latest Medical Technology - UCLA Health, Los Angeles, CA. Available at: https://www.uclahealth. org/about-us. Accessed November 29, 2018
19 Martin JA, Hamilton BE, Osterman MJK. Births in the United States, 2016. NCHS Data Brief 2017;(287):1-8

20 Athena Research Study. The Current State of Patient Portal Adoption. Athena Health. Available at: https://www.athenahealth.com/blog/2015/07/30/athenaresearch-study-the-currentstate-of-patient-portal-adoption. Published July 30, 2015. Accessed November 20, 2018

21 Elston Lafata J, Miller CA, Shires DA, Dyer K, Ratliff SM, Schreiber M. Patients' adoption of and feature access within electronic patient portals. Am J Manag Care 2018;24(11):e352-e357

22 Ali SB, Romero J, Morrison K, Hafeez B, Ancker JS. Focus section health IT usability: applying a task-technology fit model to adapt an electronic patient portal for patient work. Appl Clin Inform 2018;9(01):174-184

23 Walker DM, Menser T, Yen P-Y, McAlearney AS. Optimizing the user experience: identifying opportunities to improve use of an inpatient portal. Appl Clin Inform 2018;9(01):105-113

24 Hefner JL, Sieck CJ, McAlearney AS. Training to optimize collaborative use of an inpatient portal. Appl Clin Inform 2018;9(03): 558-564

25 Wieland D, Gibeau A, Dewey C, Roshto M, Frankel H. Patient portal readiness among postpartum patients in a safety net setting. Appl Clin Inform 2017;8(03):698-709

26 Griffin A, Skinner A, Thornhill J, Weinberger M. Patient portals: who uses them? What features do they use? And do they reduce hospital readmissions?. Appl Clin Inform 2016;7(02): 489-501 